\title{
Effects of mesh density on LES of the flow structure in internal
} \section{combustion engine}

\author{
D. TANG ${ }^{1} \&$ Z.H. ZHU ${ }^{1}$ \& L.F. SHEN ${ }^{1} \&$ B. Zhang ${ }^{2}$ \\ ${ }^{1}$ School of Automobile and Traffic Engineering, Jiangsu University, Zhenjiang, China \\ ${ }^{2}$ Wuxi South Ocean college, Wuxi, China
}

KEYWORD: diesel engine; flow structure; large-eddy simulation; mesh density ABSTRACT: By using the software FIRE, the flow structure of 186FA diesel engine were analyzed by numerical simulation. The indicator diagram, velocity field and turbulent kinetic energy in the cylinder were simulated by both the $\mathrm{k}-\varepsilon$ model and the large-eddy simulation(LES) model. The results shows that the mesh density has little effect on the flow structure when the $\mathrm{k}-\varepsilon$ model is adopted. Compared with the $\mathrm{k}-\varepsilon$ model, the LES model shows a significantly different and relatively more complex turbulence structure, and its turbulent kinetic energy is much lower than that of the latter. At the same time, the finer the mesh is, the more distinct the effect is.

\section{introduction}

Among the turbulence models, Navier-Stoke (RANS) model was widely used in the numerical simulation of the in-cylinder flow field in the previous studies. In recent years, the large-eddy simulation model (LES) of internal combustion engines is carried out abroad ${ }^{[1 \sim 4]}$. Nation ${ }^{[5]}$ originally applied the LES model into the turbulence of engine, then LES methods and its application in the complex flow of the internal combustion engine were systematically and comprehensively studied by Haworth ${ }^{[6]}$, and the results are obviously improved compared with those of various RANS models. Nevertheless, researchs of large eddy simulation have little achievements in China, Xie Maozhao ${ }^{[7]}$ have made lots of researchs on the large eddy simulation of turbulence characteristic through intake process of internal combustion engines. Recently, Qin Wenjin has studied the effect of sub-grid scale turbulence model and mesh density on the result of large eddy simulation by using the KIVALES source code ${ }^{[8]}$.

In this paper, by using the LES turbulence model of the software FIRE, the cylinder pressure, velocity field and turbulent kinetic energy of the internal combustion engine were analyzed, as well as the effect of mesh density on large-eddy simulation of the flow structure in internal combustion engine, and the result was compared with the general $\mathrm{k}-\varepsilon$ model in RANS model.

\section{calculation models}

By using the software FIRE, the flow structure of 186FA diesel engine were analyzed by numerical simulation. The basic parameters of the engine are as follows: 4-stroke, air-cooled, direct injection and diesel engine Bore×stroke: $86 \times 72 \mathrm{~mm}$, rated power: $6.0 \mathrm{~kW} / 3600 \mathrm{rpm}$.

The calculation interval is selected from the intake valve closing $\left(224^{\circ} \mathrm{CA}\right)$ to the exhaust valve opening $\left(484^{\circ} \mathrm{CA}\right)$, and the initial pressure and the temperature of the cylinder are assumed as uniformity, then the data were obtained from the experiment. According to the experimental data, fuel injection time was set at $351{ }^{\circ} \mathrm{CA}$, and the fuel injection duration was set at $20^{\circ} \mathrm{CA}$.

As shown in Figure 1, at $400^{\circ} \mathrm{CA}$, two different mesh densities were used in the calculation. Figure 1a shows a general mesh, its grid number is 119304 . As can be seen in Figure 1b, it is a fine mesh, and the grid number is 1091664 . 


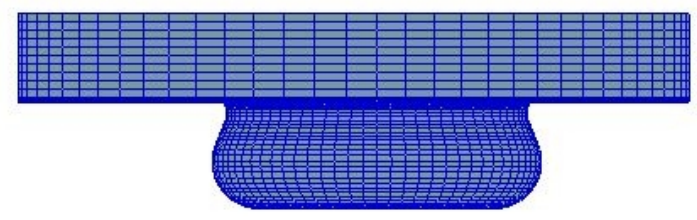

(a) General mesh

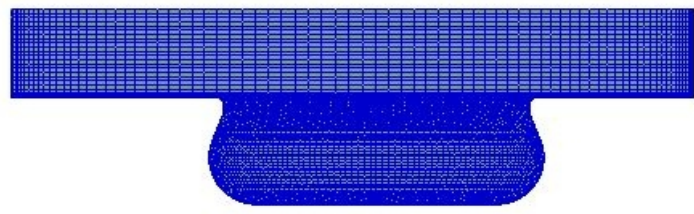

(b) Fine mesh

Fig.1 Calculational meshs

\section{calculation results and analysis}

Under the operating condition as $100 \%$ loads, 3600r/min, two kinds of models and two kinds of meshs were respectively used to simulate the indicator diagram, and the result was compared with the experimental data. At the same time, the velocity field and turbulent kinetic energy at several specific crankshaft angle were analyzed.

\section{Indicator diagram analysis}

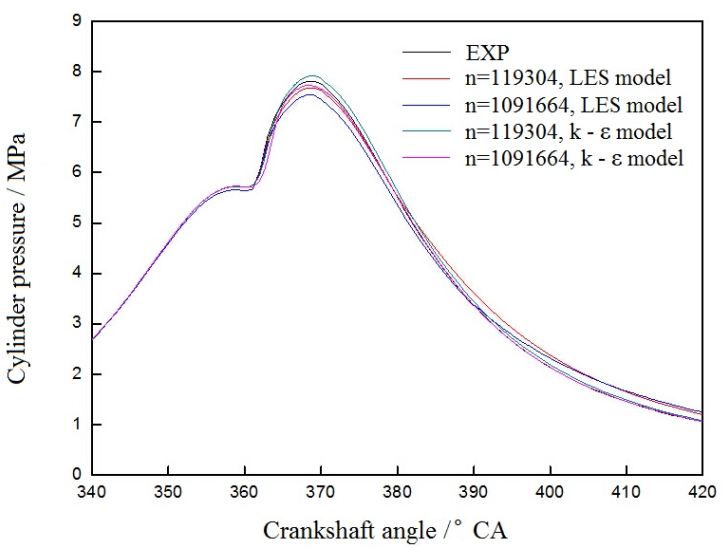

Fig.2 Indicator diagram

Figure 2 shows the two turbulence simulated results are basically fit the experimental data, which verify the rationality of the chosen models. The maximum explosion pressure of the experimental data is $7.81 \mathrm{MPa}$, the $\mathrm{k}-\varepsilon$ model shows that the maximum explosion pressure of the original mesh is $7.91 \mathrm{MPa}$, and the maximum explosion pressure of the refinement mesh is 7.72MPa. The error range of the maximum explosion pressure is less than $2 \%$, so it shows that the accuracy of the average pressure will not change significantly, even if the refined density is changed greatly.

For the LES model, the simulation result shows that the maximum explosion pressure of the original mesh is $7.68 \mathrm{MPa}$, and the maximum explosive pressure of the refinement mesh is $7.28 \mathrm{MPa}$. The error between the maximum explosive pressure of the refinement mesh and the experimental data reached to $7 \%$. Thus, for the LES model, the peak pressure of the indicator diagram changes 
obviously with the mesh density.

The simulation is based on Lenovo 24-Core C30 workstation as the hardware resources. In the general mesh, it takes about 6 hours to use the $\mathrm{k}-\varepsilon$ model, and takes about 4 hours in LES model; In the fine mesh, using the $\mathrm{k}-\varepsilon$ model takes about 68 hours, and LES takes about 41 hours. For the $\mathrm{k}-\varepsilon$ model, when the mesh density increased, the accuracy of the calculation raised little, but the calculation scale increased obviously. Therefore, it is important to consider the turbulence model and the calculation time when determining the size of the mesh element for the mesh density directly affects the size of the calculation scale.

\section{Velocity field analysis}

Figure 3 and Figure 4 are the distribution of the velocity field respectively calculated by two kinds of models and two kinds of meshs. As can be seen in Figure 3b、 Figure 3d、 Figure 4b and Figure $4 \mathrm{~d}$, when using the $\mathrm{k}-\varepsilon$ model, the fine degree of the mesh has little effect on the structure of the flow field. The main reason is that the velocity field of RANS is average calculated during a period of time and wiped out the pulsation data.

As can be seen in Figure 3a、 Figure 3b、 Figure 4a and Figure 4b, when using the same mesh precision, LES model shows a more complex turbulence structure, and the finer the mesh is, the more complex the flow field is. When using the $\mathrm{k}-\varepsilon$ model, eddies are larger and the velocity field is uniform in the center of the combustion chamber. However, in LES model, the small eddy structure appeared in the corresponding position, and the turbulence characteristics were more obvious. The reason is that the velocity field of LES model shows the turbulence filtered by mesh, it filtered the small eddy pulsation but retained the large eddy pulsation.

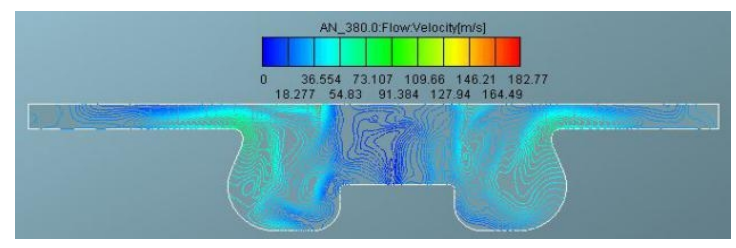

(a)n=119304, LES model

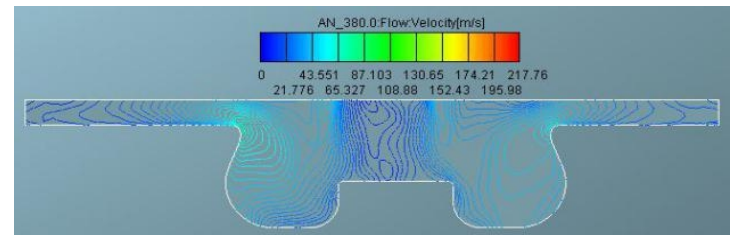

(b) $\mathrm{n}=119304, \mathrm{k}-\varepsilon$ model

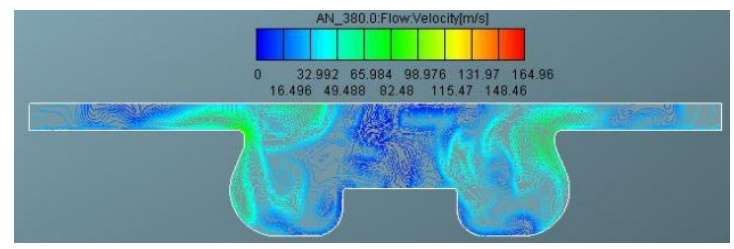

(c)n=1091664, LES model

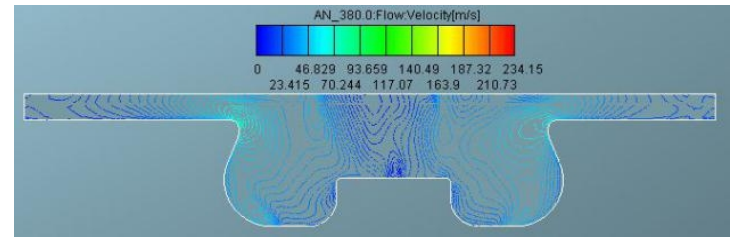

(d)n=1091664, k - $\varepsilon$ model

Fig. 3 Distribution of the velocity field at $380^{\circ} \mathrm{CA}$ 


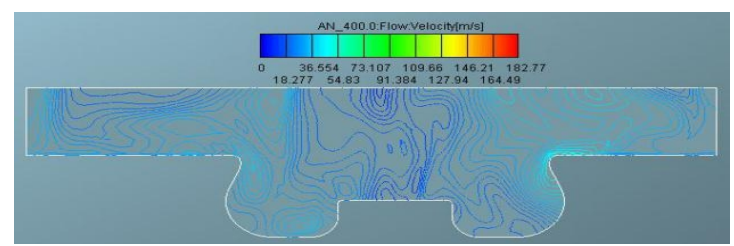

(a)n=119304, LES model

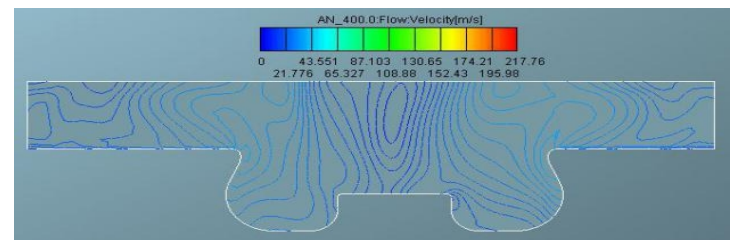

(b)n=119304, k - $\varepsilon$ model

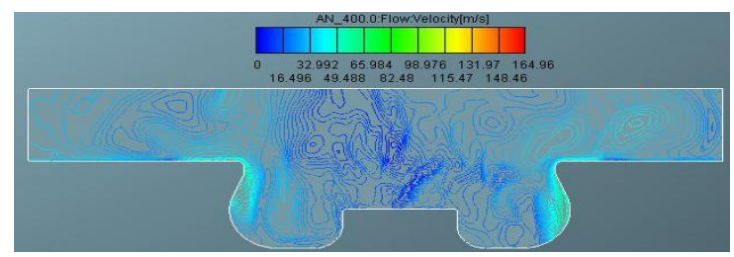

(c)n=1091664, LES model

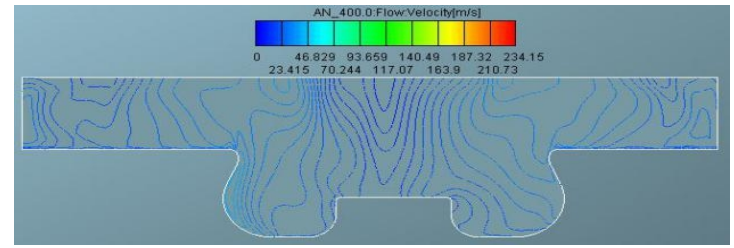

(d)n=1091664, k - $\varepsilon$ model

Fig.4 Distribution of the velocity field at $400^{\circ} \mathrm{CA}$

\section{Turbulent kinetic energy analysis}

Figure 5 and Figure 6 are the distribution of the turbulent kinetic energy respectively calculated by two kinds of models and two kinds of meshs. It can be seen that the turbulent kinetic energy calculated by LES is much lower than the $\mathrm{k}-\varepsilon$ model, the main reason is the turbulent kinetic energy calculated by the $\mathrm{k}-\varepsilon$ model include the energy of all the eddies average calculated during a period of time. However, the turbulent kinetic energy calculated by LES model is the energy of small eddies filtered by the mesh, that is to say, LES model can capture small eddies with smaller turbulent kinetic energy. At the same time, it can be seen that the turbulent kinetic energy calculated by LES is more uniform than that calculated by $\mathrm{k}-\varepsilon$ model in the center of the cylinder. Nevertheless, it is opposite at the bottom of the complex pit and the top edge of the piston, which illustrate that these regions are gathered by the small eddies.

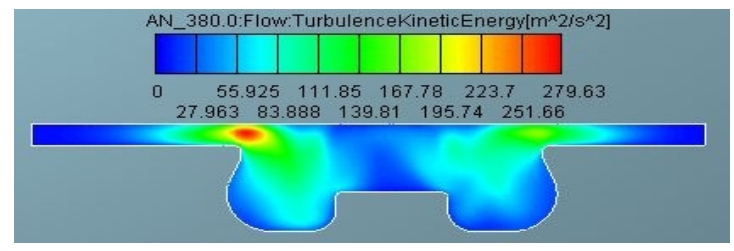

(a)n=119304, k - $\varepsilon$ model 


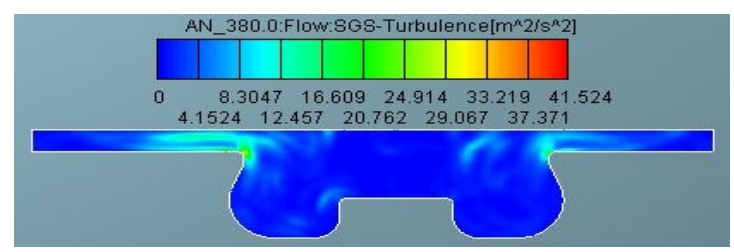

(b) $n=119304$, LES model

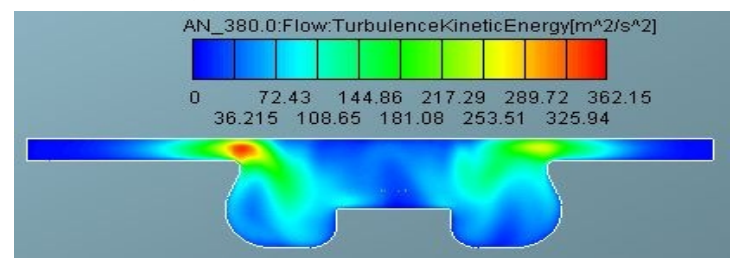

(c)n=1091664, k - $\varepsilon$ model

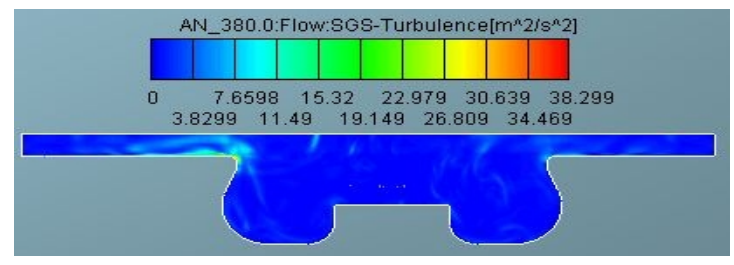

(d)n=1091664, LES model

Fig. 5 Distribution of the turbulent kinetic energy at $380^{\circ} \mathrm{CA}$

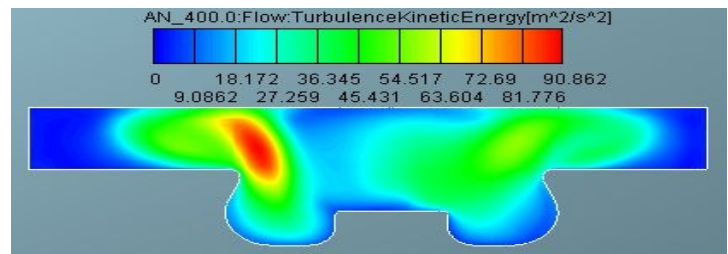

(a)n=109304, k - $\varepsilon$ model

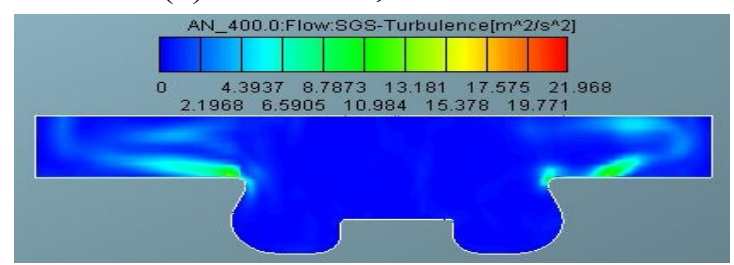

(b) $n=119304$, LES model

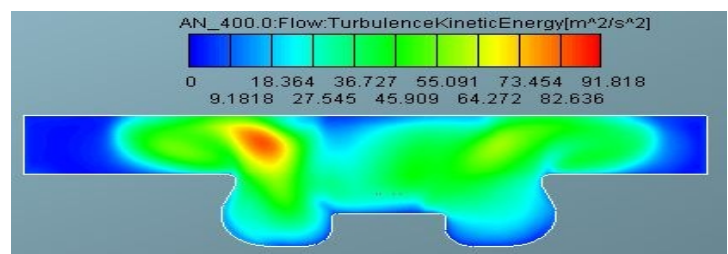

(c)n=1091664, k - $\varepsilon$ model

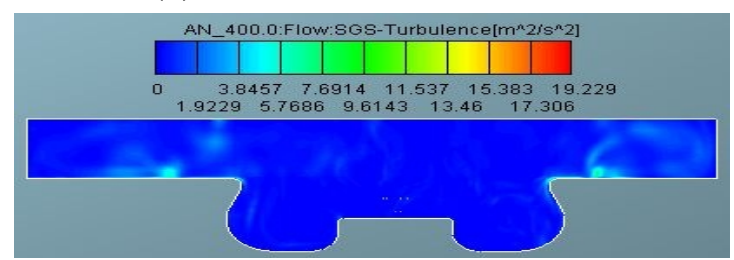

(d)n=1091664, LES model

Fig.6 Distribution of the turbulent kinetic energy at $400^{\circ} \mathrm{CA}$ 


\section{Conclusions}

(1) The change of mesh density has little effect on the cylinder pressure of the RANS, but it has a great effect on the peak cylinder pressure changes of the LES.

(2) For the LES model, the finer the mesh is, the finer eddies can captured, which can show the advantages of the model. However, when the $\mathrm{k}-\varepsilon$ model meets the precision requirement, even if the mesh refined constantly, the accuracy can't be significantly improved, and the time will be greatly increased.

(3) Compared with the $\mathrm{k}-\varepsilon$ model, the LES model shows a significantly different and relatively more complex turbulence structure. Furthermore, the LES model can capture a wider range of turbulence structure than the $\mathrm{k}-\varepsilon$ model, and it shows a finer turbulence structure.

\section{ACKNOWLEDGEMENTS}

This work was financially supported by the Priority Academic Program Development (PAPD), Open Fund of the state key Laboratory of internal combustion engines (NO.K2014-9) in Tianjin university of China.

\section{Reference}

[1]Jhavar, R. \& Rutland, C. 2006. Using Large Eddy Simulations to Study Mixing Effects in Early Injection Diesel Engine Combustion. SAE Technical Papers: 2006-01-0871

[2]Hu, B. \& Rutland, C. Flamelet Modeling with LES for Diesel Engine Simulations. SAE Technical Papers: 2006-01-0058

[3]Huijinen, V \& Somers, L.M.T. \& Baert, R.S.G. 2005. Validation of the LES Approach in Kiva $-3 \mathrm{~V}$ on a Square Duct Geometry. International Journal for Numerical Methods in Engineering 64: 907-919

[4]Lee, D. \& Pomraning, E. \& Rutland, C. 2002. LES Modeling of Diesel Engines. SAE Technical Papers: 2002-01-2779

[5]Nation, K. \& Kuwahara ,K. 1992. Large eddy simulation and direct simulation of compressible turbulence and combusting flows in engines based on the BI-SCALES method. Fluid Dynamics Research 10: 299-325.

[6]Haworth, D.C. 1994. Large-eddy simulation of in-cylinder flows. Oil \& Gas Science and Technology 54(2): 175-185

[7]Yang, P \& Xie M.Z 2008 Large eddy simulation of turbulence characteristic through intake process of internal combustion engines. Dalian University of Technology : 1-80

[8]Qin, W.J \& Xie, M.Z \& Jia, M 2011. Large eddy simulation of turbulent flows and cyclic varations in internal combustion engine. Journal of Engineering Thermophysics (s1): 268-271 